\title{
Turbulence Production by Rough Boundaries
}

\author{
Richard I Leighton* \\ University of Michigan, Ann Arbor, MI 48109
}

\begin{abstract}
The effects of roughness in an incompressible turbulent boundary layer include the increased production of turbulence kinetic energy (TKE) and altered the nature of the skin drag. By formulating the exact Reynolds averaged Navier-Stokes and the turbulence kinetic energy equations in a manner that includes an arbitrary roughness, the averaged terms representing the roughness production of TKE and the roughness drag can be written explicitly. These terms are calculated from a collection of direct numerical simulations (DNS) wherein the roughness geometry is represented using the immersed boundary methodology. The roughness geometry is limited to a simple array of vertically oriented cylinders in these simulations. The results include an examination of the partitioning of the production of TKE into canonical shear production and production by roughness, and the partitioning of drag into form drag and viscous shear drag. The relevance of the partioning will be discussed in the context of turbulence modeling
\end{abstract}

\section{Nomenclature}

$\begin{array}{ll}k^{+} & \text {Roughness Height (viscous lengths) } \\ k & \text { Turbulence Kinetic Energy } \\ \epsilon & \text { dissipation } \\ <-> & \text { Weighted Averaging Operator } \\ H & \text { Indicator Function or channel half width } \\ \beta=<H> & \text { Void Fraction } \\ \bar{\phi}=\frac{<H \phi>}{\beta} & \text { Phase Average } \\ U & \text { Velocity } \\ P & \text { Pressure } \\ U^{+}=U / u_{\tau} & \\ \Delta U^{+} & \text {Hama Roughness Function } \\ \tau_{w}, \tau_{\text {boundary }} & \text { Wall Shear Stress } \\ \tau_{r, i j} & \text { Effective Roughness Stress } \\ x_{s} & \text { Rough Surface Location } \\ n_{j} & \text { Rough Surface normal vector } \\ u_{\tau}=\sqrt{\tau_{w} / \rho} & \text { friction velocity } \\ l^{+}=\nu / u_{\tau} & \text { viscous length } \\ \text { Subscript } & \\ r & \\ i & \text { Roughness }\end{array}$

\section{Introduction}

The development of smooth wall turbulence models has benefitted for data derived from direct numerical simulations (DNS) by allowing the detailed examination of the processes to be modeled. A set of DNS results covering a range of roughness parameter range would be useful in examining the role played by the roughness

\footnotetext{
*Adjunct Research Associate, Dept of Naval Architecture and Marine Engineering.
} 
elements in the generation of both form drag, turbulence kinetic energy (TKE) and Reynolds shear stress. The inability to find appropriate data for model development led to this effort.

The effects of wall roughness have been calculated via direct numerical simulations by several groups. Two general approaches are in these simulations. Ikeda and Durbin ${ }^{1}$ used a numerical scheme wherein the no-slip boundary conditions are explicitly enforced on the boundary. They considered the transverse rib geometry used in Hanjalic and Launder. ${ }^{2}$ Unfortunately, they only simulate two conditions, too limited to for use in understanding the scaling behavior of roughness. The immersed boundary technique is the other general approach employed for roughness simulations. In immersed boundary simulations, represented by the work of Bhagananar, Kim and Coleman, ${ }^{3}$ a force distribution is used enforce the no-slip condition on an arbitrary surface within the computational domain. Again, although many simulations have been performed, they have not been directed at the development of turbulence models.

The development of turbulence models for rough walled flows has generally followed two approaches. The first approach initally suggested by Schlichting, ${ }^{4}$ referred to as discrete roughness modeling and exemplified by the work of Taylor ${ }^{5}$ models the induced roughness drag in the momentum equation by considering the geometric shape of the elements and employing an appropriate drag coefficient. Typically, the impact of the roughness on the turbulent kinetic energy is not included. In contrast, in the eddy viscosity modeling approach the direct effects of form drag are ignored and the emphasis is placed on the capturing the appropriate behavior using an augmented eddy viscosity. One of the earliest models of this type is the mixing length model of Cebecci and Chang ${ }^{6}$ in which the mixing length is made dependent of the roughness and the eddy viscosity is defined to be non-zero at the wall. Durbin, et al. ${ }^{7}$ combined several approaches in the development of an updated roughness model.

A more consistent $k-\epsilon$ roughness was developed by Leighton and Walker. ${ }^{8}$ They started from the exact Reynolds averaged equations, described below, in which the effects of roughness are explicitly represented. They assumed that the details of the form drag could be ignored and that the neglected form drag would be captured by increased viscous wall shear stress. A full Reynolds stress tensor model was developed and contracted into the $k-\epsilon$. While the model presented performed very well, several assumptions made during development could not be tested. Therefore in this work, the following issues will be examined:

Form drag Is the assumed stress decomposition in which the form drag is absorbed into the viscous shear appropriate?

Roughness Production What is the magnitude and distribution of the roughness induced production of turbulence kinetic energy?

\section{Reynolds-Averaged Governing Equations}

To exploit DNS data for model development, a set of Reynolds-averaged governing equations need to be developed. Two forms of the averaged governing equations can be developed depending how the averaging is done and on how the roughness is represented. In the two-phase formulation, an indicator function is used to distinguish between the solid (roughness elements) and the fluid regimes prior to averaging (See Joseph, ${ }^{9}$ Leighton and Walker ${ }^{8}$ ). The boundary forcing is naturally included as an integral capturing the hydrodynamic forcing acting on the arbitrarily rough boundary. This form is the natural setting in which to develop turbulence models. However, to use this representation in evaluating the mean forcing requires knowing the instantaneous stress on the arbitrary, but fixed, boundary. Such a formulation would be appropriate for the simulations of Ikeda and Durbin ${ }^{1}$ where the boundaries are explicitly represent.

For simulations using the immersed boundary formulation, the immersed boundary force is distributed in three dimensions, explicitly known and available during the averaging process. Formulation of the averaged equations follows directly from the standard approach. It is important to note that these averaged equations

reduce to their smooth wall counterpart at location removed from the roughness. The final form of these averaged equation is identical to the canonical form, though some interesting comments can be made about the boundary force term in the TKE equation.

\section{II.A. Two-phase roughness representation}

The transport equations for the mean velocity, the turbulent stresses and the temperature field can be derived in a manner that includes the wall roughness geometry by adopting a multiphase averaging approach. In

2 of 10

American Institute of Aeronautics and Astronautics 
this approach, an indicator function, H, defined an one in the fluid phase and zero in the solid phase, is used as a weighting factor in the averaging process. The averaging process is defined over both space and time $\left(\right.$ Drew $\left.^{10}\right)$. As a consequence of this approach, the roughness contributes to an interfacial source term distributed in space. Drew developed the approach in the context of multiphase flow, but as noted by Joseph, ${ }^{9}$ this approach is applicable even when the second species is a solid.

The phase-averaged Reynolds averaged Navier-Stokes equation can be written as:

$$
\rho_{f} \frac{\partial\left(\beta \overline{\mathrm{U}}_{i}\right)}{\partial \mathrm{t}}+\rho_{f} \frac{\partial}{\partial x_{j}}\left[\beta \overline{\mathrm{U}}_{\mathrm{f} i} \overline{\mathrm{U}}_{\mathrm{f} j}\right]=-\rho_{f} \frac{\partial}{\partial x_{j}}\left\langle\mathrm{Hu}_{\mathrm{f}_{i} \mathrm{u}_{\mathrm{f}}}\right\rangle-\frac{\partial \beta \overline{\mathrm{P}_{\mathrm{f}}}}{\partial \mathrm{x}_{\mathrm{i}}}+\mu_{f} \frac{\partial^{2}\left(\beta \overline{\mathrm{U}}_{i}\right)}{\partial \mathrm{x}_{\mathrm{j}}{ }^{2}}-\left\langle\mathrm{T}_{i j} n_{j} \delta\left(x-x_{s}\right)\right\rangle,
$$

where $\langle>$ is the averaging operator and $\beta=<\mathrm{H}>$ is the fluid fraction. The first term on the right-hand-side of the RANS equations is a Reynolds stress term resulting from averaging the convective nonlinearity. This term reverts to the canonical Reynolds stress whenever $\beta$ is unity. The second and third terms are similar to the single-phase counterparts. The fourth term is the mean interfacial stress term. The term is the average of inner product of the wall stress, $\mathrm{T}_{i j}$, and the normal vector, $n_{j}$, acting at the wall location, $\delta\left(x-x_{s}\right)$. In the case of a smooth-wall no-slip boundary, this term represents the stress applied to the fluid at the boundary to balance the viscous shear at the boundary. For a rough wall, this term is a distributed force acting of the fluid representing the average of the wall stress over a distribution of wall locations.

The phase-averaged turbulence kinetic energy (TKE) equation is

$$
\begin{aligned}
& \frac{\rho}{2}\left[\frac{\partial}{\partial \mathrm{t}}+\overline{\mathrm{U}}_{\mathrm{f}_{j}} \frac{\partial}{\partial \mathrm{x}_{\mathrm{j}}}\right]\left(\frac{\left\langle\mathrm{Hu}_{\mathrm{f}_{i}} \mathrm{u}_{\mathrm{f} i}\right\rangle}{\beta}\right)=-\frac{\rho}{2 \beta} \frac{\partial}{\partial \mathrm{x}_{\mathrm{j}}}\left\langle\mathrm{Hu}_{\mathrm{f}_{j}} \mathrm{u}_{\left.\left.\mathrm{f}_{i} \mathrm{u}_{\mathrm{f}}\right\rangle\right)}{ }_{\text {turb diff }}\right. \\
& -\frac{\rho_{f}}{\beta}\left\langle\mathrm{Hu}_{\left.\mathrm{f}_{i} \mathrm{u}_{\mathrm{f}}\right\rangle \frac{\partial \overline{\mathrm{U}}_{\mathrm{f} i}}{\partial \mathrm{x}_{\mathrm{j}}}}{ }_{\text {Prod }}-\frac{1}{\beta} \frac{\partial}{\partial \mathrm{x}_{\mathrm{k}}}\left\langle\mathrm{Hu}_{\mathrm{f} k} \mathrm{p}_{\mathrm{f}}\right\rangle{ }_{\text {press diff }}+\frac{\mu_{f}}{\beta} \frac{\partial^{2}}{\partial \mathrm{x}_{\mathrm{j}}{ }^{2}}\left[\frac{\left\langle\mathrm{Hu}_{\mathrm{fi}} \mathrm{u}_{\mathrm{f} i}\right\rangle}{2}\right]{ }_{\text {viscous diff }}\right.
\end{aligned}
$$

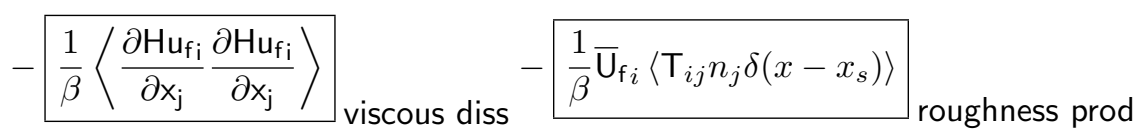

where the processes represented by the terms on the right hand side are identified. Removed from the rough boundary, where the fluid fraction is identically one, these terms revert to their canonical forms, including the term identified as roughness production. The roughness production term is zero at all points where the fluid fraction is unity. Likewise for a smooth no-slip boundary, the roughness production is zero (on boundary). However, for a rough boundary, this term is a source of turbulent kinetic energy.

\section{II.B. For an immersed boundary representation}

In the immersed boundary representation the boundary force representing the effects of the roughness are known explicitly from the calculation. The imposed force can be decomposed into a mean and fluctuation contribution, $f_{r_{i}}=\left(f_{r_{i}}^{\prime}+\overline{\mathrm{f}}_{\mathrm{r}_{\mathrm{i}}}\right)$ and the Reynolds-averaged Navier-Stokes equations can be formed in the usual manner:

$$
\frac{\partial \overline{\mathrm{U}}_{\mathrm{i}}}{\partial \mathrm{t}}+\overline{\mathrm{U}}_{\mathrm{j}} \frac{\partial \overline{\mathrm{U}}_{\mathrm{i}}}{\partial \mathrm{x}_{\mathrm{j}}}+\frac{\partial \overline{\mathrm{u}_{\mathrm{j}} \mathrm{u}_{\mathrm{i}}}}{\partial \mathrm{x}_{\mathrm{j}}}=-\frac{1}{\rho} \frac{\partial \overline{\mathrm{P}}}{\partial \mathrm{x}_{\mathrm{i}}}+\nu \frac{\partial^{2} \overline{\mathrm{U}}_{\mathrm{i}}}{\partial \mathrm{x}_{\mathrm{k}}^{2}}+\overline{\mathrm{f}}_{\mathrm{r}}
$$

The TKE equations, constructed in the usual way can be rewritten as:

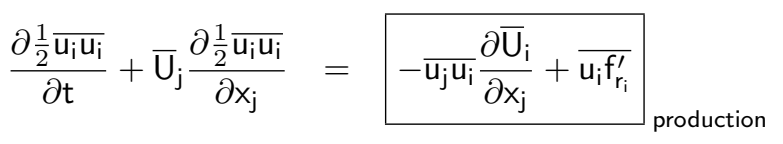

$$
\begin{aligned}
& -\frac{\partial \frac{1}{2} \overline{\mathrm{u}_{\mathrm{i}} \mathrm{u}_{\mathrm{i}} \mathrm{u}_{\mathrm{j}}}}{\partial \mathrm{x}_{\mathrm{j}}}-\frac{1}{\rho} \frac{\partial \overline{\mathrm{u}_{\mathrm{i}} \mathrm{p}}}{\partial \mathrm{x}_{\mathrm{i}}}+\nu \frac{\partial^{2} \frac{1}{2} \overline{\mathrm{u}_{\mathrm{i}} \mathrm{u}_{\mathrm{i}}}}{\partial \mathrm{x}_{\mathrm{k}}{ }^{2}}{ }_{\text {diffusion }} \\
& -\nu \overline{\frac{\partial \mathrm{u}_{\mathrm{i}}}{\partial \mathrm{x}_{\mathrm{k}}} \frac{\partial \mathrm{u}_{\mathrm{i}}}{\partial \mathrm{x}_{\mathrm{k}}}}{ }_{\text {dissipation }},
\end{aligned}
$$


where $f_{r_{i}}^{\prime}$ is the production of turbulence kinetic energy as a result of the roughness.

A limitation of the immersed boundary approach, as implemented below, can be seen in the development of the roughness production term. Equation II.B is formed by subtracting the equation for the kinetic energy of the mean flow from the equation for the total kinetic energy. The correlation between the immersed boundary force and the instantaneous velocity occurs in the equation for the total kinetic energy. This term can be written as:

$$
\begin{aligned}
\left(\mathrm{u}_{\mathrm{i}}+\overline{\mathrm{U}}_{\mathrm{i}}\right) \times\left(\mathrm{f}_{\mathrm{r}_{\mathrm{i}}}^{\prime}+\overline{\mathrm{f}}_{\mathrm{r}_{\mathrm{i}}}\right) & = \\
\mathrm{u}_{\mathrm{i}} \mathrm{f}_{\mathrm{r}_{\mathrm{i}}}^{\prime}+\mathrm{u}_{\mathrm{i}} \overline{\mathrm{f}}_{\mathrm{r}_{\mathrm{i}}}+\overline{\mathrm{U}}_{\mathrm{i}} \mathrm{f}_{\mathrm{r}_{\mathrm{i}}}^{\prime}{ }_{\mathrm{A}}+\overline{\mathrm{U}}_{\mathrm{i}} \overline{\mathrm{f}}_{\mathrm{r}_{\mathrm{i}}} & =\mathrm{E}_{\mathrm{f}}
\end{aligned}
$$

The term in the box marked A will be zero after averaging. Note that if the rough surface is a true non-slip surface, the force $\left(f_{r_{i}}^{\prime}+\overline{\mathrm{f}}_{\mathrm{r}_{\mathrm{i}}}\right)$ will exist only on the rough boundary, but the velocity on the boundary

will be zero, $\left[u_{i}+\bar{U}_{i}\right]_{\text {bndy }}=0$. Therefore, if the boundary is a no-slip, then $E_{f}=0$. Like turbulent shear stresses, roughness does not generate turbulence, but extracts energy from the mean flow, transferring it to the turbulent flow. Under the conditions of the pseudo-roughness, the boundary is not a perfect no-slip boundary and $E_{f} \neq 0$. Current calculations show that $E_{f} \leq 0$. Since the mean force imposed by the roughness is acting against the mean velocity, the term

$$
\overline{\mathrm{U}}_{\mathrm{i}} \overline{\mathrm{f}}_{\mathrm{r}_{\mathrm{i}}} \leq 0 \text {. }
$$

Then, the forcing term in the TKE equations can be rewritten as:

$$
\overline{u_{i} f_{r_{i}}^{\prime}}=E_{f}-\bar{U}_{i} \bar{f}_{r_{i}} \geq 0 .
$$

if equation 10 is substituted into the TKE equation, $\bar{U}_{\mathrm{i}} \overline{\mathrm{f}}_{\mathrm{r}_{\mathrm{i}}}$ is the energy extracted from mean flow and $\mathrm{E}_{\mathrm{f}}$ is effectively a immersed boundary-induced dissipation term.

The fact that in real flows $\overline{\mathrm{u}_{\mathrm{i}} \mathrm{f}_{\mathrm{r}_{\mathrm{i}}}^{\prime}}=-\overline{\mathrm{U}}_{\mathrm{i}} \overline{\mathrm{f}}_{\mathrm{r}_{\mathrm{i}}}$ is important in the context of turbulence model development. If the equation for the transport of TKE were formulated using equation 10 it would correspond more closely to the TKE equation derived via the two-phase formulation. Then a model for the mean external force, which is dependent on the distance from the wall, could be used in both the Reynolds-averaged Navier-Stokes equation and the turbulence kinetic energy equation.

\section{The numerical simulations}

To obtain DNS data, an existing pseudo-spectral channel flow algorithm has been extended to include an immersed boundary forcing term. A force, $\mathbf{f}_{\mathbf{r}}$, is applied at the immersed boundary in a manner to drive the velocity on the immersed boundary to zero creating a rough no-slip boundary. The approach adopted follows that of Goldstein et al., ${ }^{11}$ in which the immersed force has a slow term proportional the time-integrated (mean) velocity and a fast term proportional to the instantaneous velocity both evaluated at the virtual no-slip surface. To prevent the formation of non-local forcing due to the gibbs phenomena, the force was explicitly spread over three grid points. This limits the formation of gibbs oscillations, but does introduce the dissipation effect mentioned above. The frequently employed higher-order spectral filtering was not used.

The pressure gradient driving the flow is held constant for all simulations. The friction velocity Reynolds number, $R e_{\tau}=316$ where the friction velocity is determined from the pressure gradient:

$$
\frac{\partial P}{\partial x} \times H=\tau_{\text {boundary }}=\rho u_{\tau}^{2},
$$

where $H$ is the channel half height. The computational domain, $3 \pi \times 2 \times 2 \pi$ in units of the channel half height, is resolved with $192 \times 128^{2}$ grid points.

\section{III.A. Roughness geometry}

Roughness is applied on both boundaries of the simple channel flow geometry. In the DNS channel flow calculations discussed here top and bottom boundaries were roughened by an array of vertically oriented stout cylinders, ranging in height from 0.0 to $66.9 l^{+}$(See Table 1). The actual shape of the roughness 
Table 1. The nine simulations performed at $R e_{\tau}=316$. The only parameter adjusted in the simulations is the roughness height.

\begin{tabular}{ccccc} 
Run number & Roughness Height & $\frac{\tau_{\text {rough }}}{\tau_{\text {wall }}}$ & $U_{m}^{\dagger}$ & $\Delta U^{+}$ \\
\hline 1 & smooth & 0.0 & 1.03 & 0.0 \\
2 & $0.9 l^{+}$ & 0.08 & 1.02 & 0.1 \\
3 & $2.4 l^{+}$ & 0.13 & 1.01 & 0.3 \\
4 & $4.7 l^{+}$ & 0.20 & 1.01 & 0.3 \\
5 & $11.5 l^{+}$ & 0.43 & 0.91 & 1.8 \\
6 & $21.2 l^{+}$ & 0.65 & 0.73 & 4.8 \\
7 & $37.8 l^{+}$ & 0.78 & 0.57 & 7.2 \\
8 & $49.1 l^{+}$ & 0.84 & 0.45 & 9.2 \\
9 & $66.9 l^{+}$ & 0.87 & 0.36 & 10.8 \\
\hline
\end{tabular}

$\dagger$ Mass averaged velocity.

elements is constrained by the need to approximate them on a coarse grid, relative to their size. They are resolved as $5 \times 5$ squares with the corners removed.

The $12 \times 8$ array of roughness elements are laid out in staggered arrangement. The layout of the elements can be seen in the plot of he instantaneous stream wise velocity is shown in figure 1 . The cylinders are $66.9 l^{+}$tall and the plane of the velocity data at a distance of $57.6 l^{+}$from the boundary. For reference, the projected area of the elements as seen from above is $8.2 \%$ of the total area of one wall of the channel.

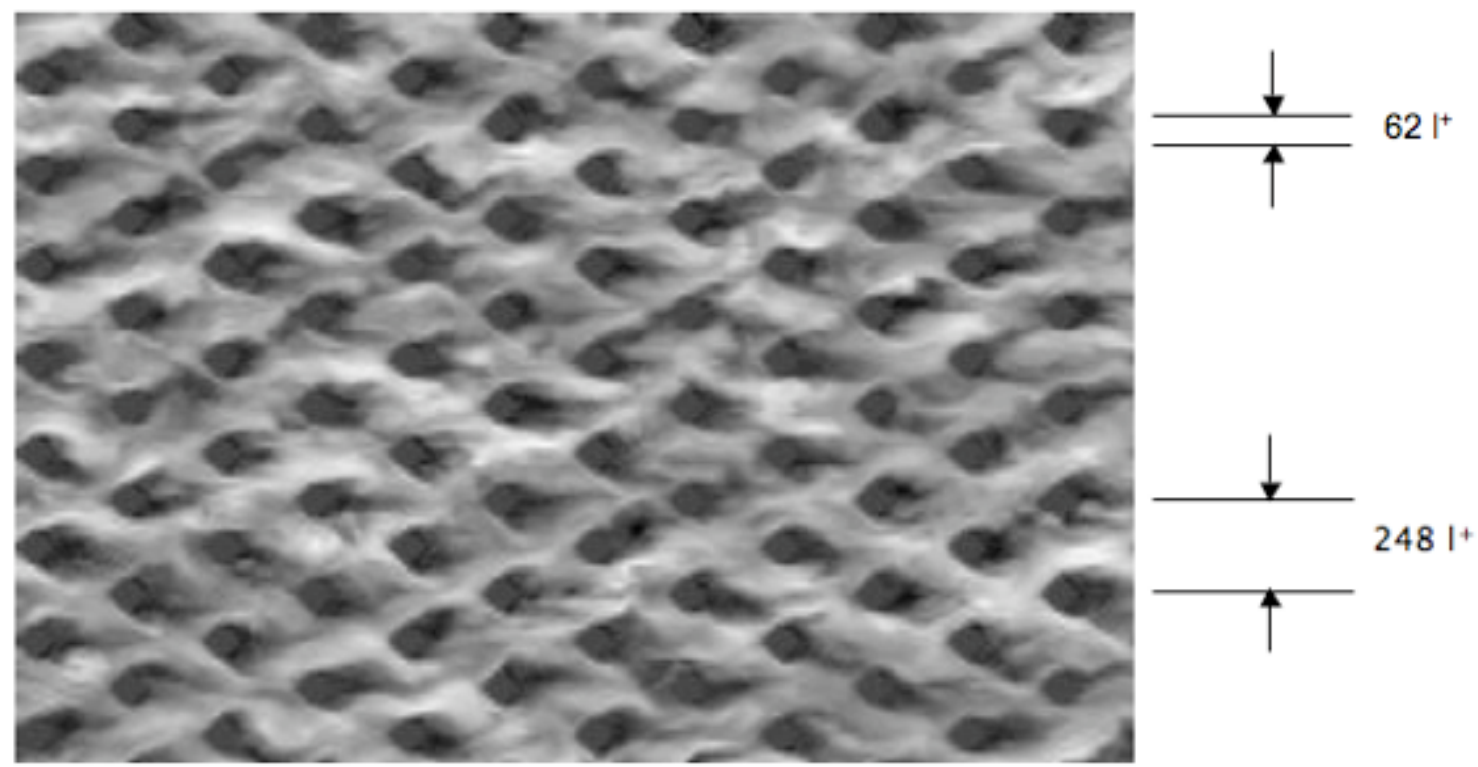

Figure 1. The streamwise velocity flowing through an array of $66.9 l^{+}$tall vertical cylinders, which are roughly $62 l^{+}$in diameter. The plane of data is a distance of $57.6 l^{+}$. The flow is from left to right. $U_{\min }=-2.4 u^{*}, U_{\max }=8.8 u^{*}$

\section{III.B. Mean Velocity}

The mean velocities decrease as expected as the roughness height increases, as can be seen in figure 2, For roughness heights increasing from 0 to $5 l^{+}$, the log layer does not change significantly. The mean velocity within $y^{+}=5$, the mean velocity drops slight as more of the drag induced by the roughness, mainly viscous shear on the tops of the roughness elements, shifts away from the wall. For roughness heights greater than $5 l^{+}$, the roughness elements are in the buffer layer of the canonical channel flow. As is evident from figure 
2, for the larger roughness elements the log layer remains defined but is shifted away from the wall.

The velocity decrement and the mass flux are plotted on the right plot of figure 2 . The velocity decrement, and hence mass flux, do not behave as would be expected for a high Reynolds number flow. For the case of high Reynolds number and for fully rough conditions, the velocity decrement is expected to behave as:

$$
\Delta U^{+}=\frac{1}{\kappa} \ln \left(k^{+}\right)-C
$$

where $C \approx 2.8$. Specifically the slope of the curve is the reciprocal of the von Karman constant. However for these simulation, which are neither high Reynolds number nor fully rough, the slope of the curve is nearly twice the expected slope. In absence of equivalent experimental results confirming or contradicting this observation, this variance is assumed to be a low Reynolds number effect.
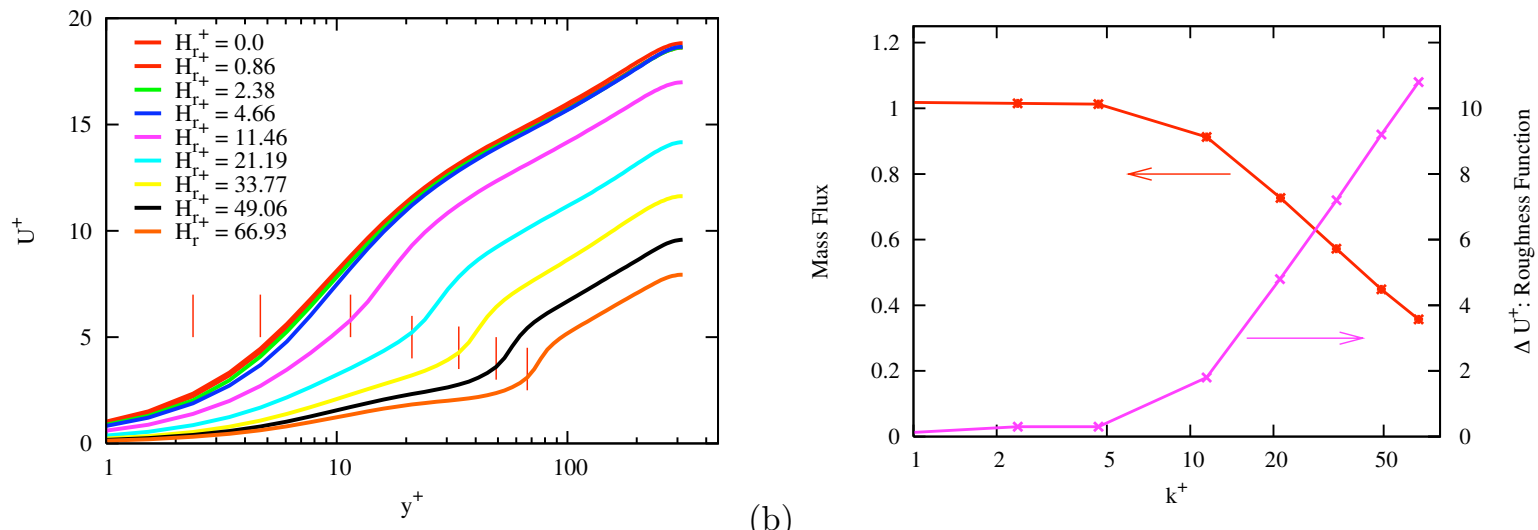

(a)

Figure 2. Mean velocity. (a) Mean velocity in log format for the nine cases. The vertical lines indicate the nominal height of the roughness elements. (b) Mass flux and roughness function as a function of nominal roughness height.

\section{III.C. Stress partioning}

The mean of the immersed boundary force which defines the roughness elements at the wall is shown in figure 3 , in the limit of the height if the roughness elements approaching zero the external force is the force required to balance the viscous wall shear stress. As the roughness height increases, the spike in the forcing presumably balancing the viscous shear on the top of the roughness element is reduced and more of the immersed boundary force is balancing the form drag.

From the data we can also calculate the partitioning of the stress into mean viscous stress, Reynolds stress and the effective stress of the mean immersed bundary force for each of the simulations:

$$
\tau_{x y}=\nu \frac{\partial U}{\partial y}-\overline{u^{\prime} v^{\prime}}+\tau_{r},
$$

where $\tau_{r}$ is the effective roughness stress. The effective roughness stress can be determined from the immersed boundary force as

$$
f_{r}=\frac{\partial \tau_{r}}{\partial y} .
$$

Equation 14 can be integrated to yield the average roughness contribution for the total shear stress balance. When normalized by the total wall stress, the stress balance determined from the momentum equation is

$$
\tau_{x y}=\nu \frac{\partial U}{\partial y}-\overline{u^{\prime} v^{\prime}}+\tau_{r}=(1-y / H) .
$$

The stress decomposition, equation 15, evaluated at the wall for the range of roughness heights is shown in figure 4. As the roughness height approaches zero, the stress carried by the roughness elements equals the viscous wall shear stress of the equivalent projected area. Therefore, for this specific geometry,

$$
\lim _{k^{+} \rightarrow 0} \frac{\tau_{r}}{\tau_{\text {wall }}} \approx 8.2 \% \text {. }
$$

$$
6 \text { of } 10
$$


Although the mass flux is effectively unaltered for $k^{+}=4.6$, the roughness elements carry $20 \%$ of the wall shear stress. As seen in figure 4 the portion of the wall shear stress carried by roughness begin to asymptote to about $90 \%$ for the largest roughness elements.

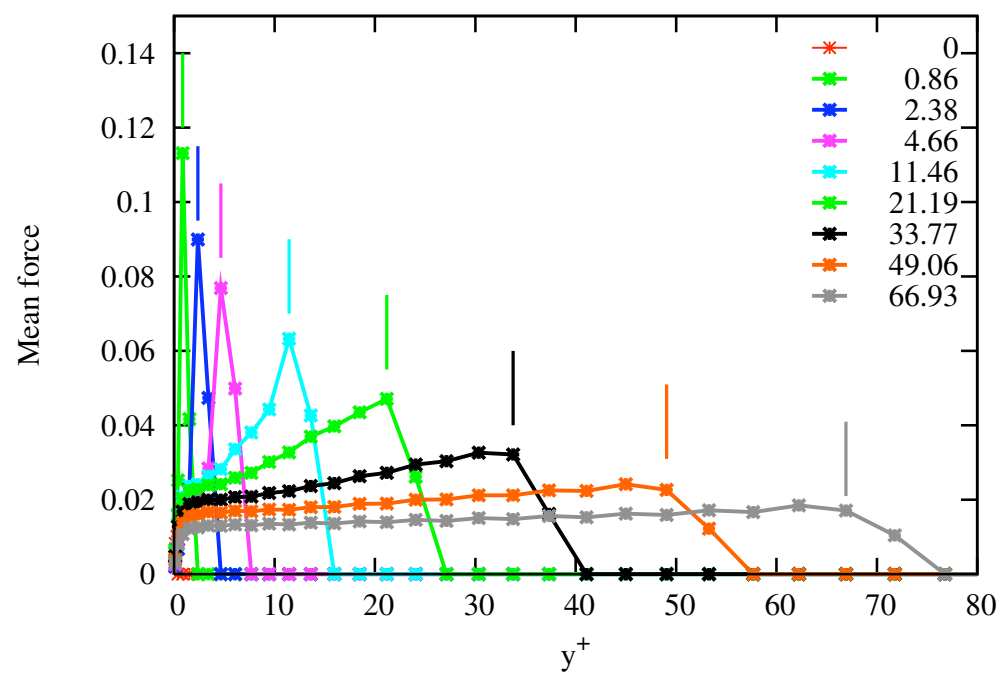

Figure 3. Mean external force. The vertical lines indicate the nominal roughness heights.

The stress decomposition across the channel is shown in figure 5. The left figure is the stress decomposition from the wall to the centerline. Sum of the stresses converges to the expected linear distribution. The same data is shown on the right side of figure 5 . With increasing roughness height, the effective roughness stress, the black curves, extend further from the wall. The viscous stress at the wall decreases with increasing roughness to balance the increasing roughness stress. The mean viscous shear stress has a local maximum at a height somewhat above the roughness elements, possibly because these are very plateau-like roughness elements. This local maximum in the viscous shear is balanced in part by a reduction in turbulent shear stress with increasing roughness height. For the roughness heights less than $k^{+}=5$, the Reynolds stress is not significantly affected. As the increasing roughness heights, the Reynolds stress decreases.

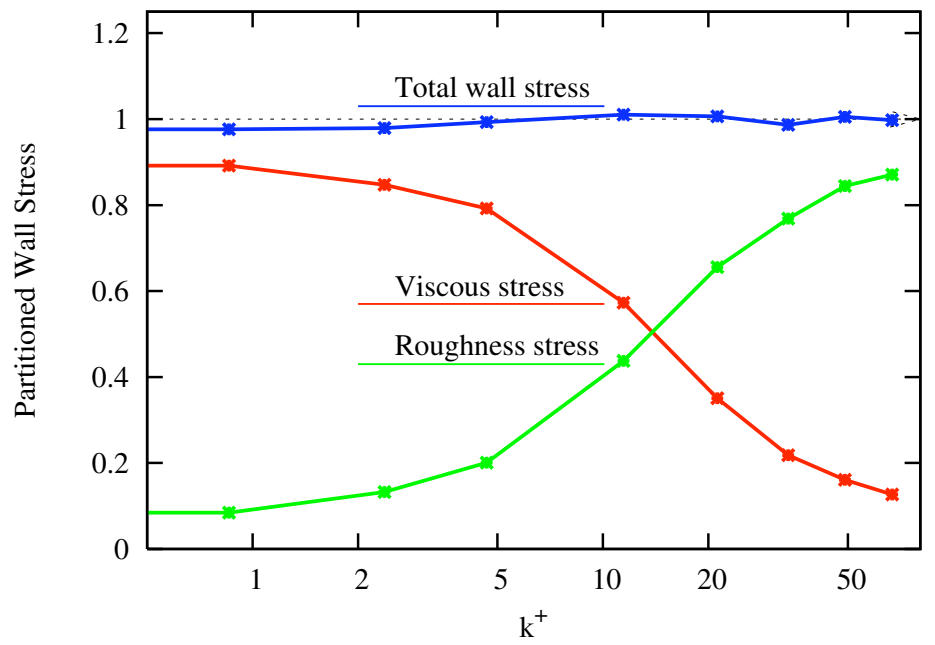

Figure 4. Effective shear stress partition at the wall. The green curve is the effective roughness-induced shear stress and the red curves are the viscous stress. The total stress in blue the the sum of these. The stresses are normalzed by the wall stress, $\tau_{\text {boundary }}$ 

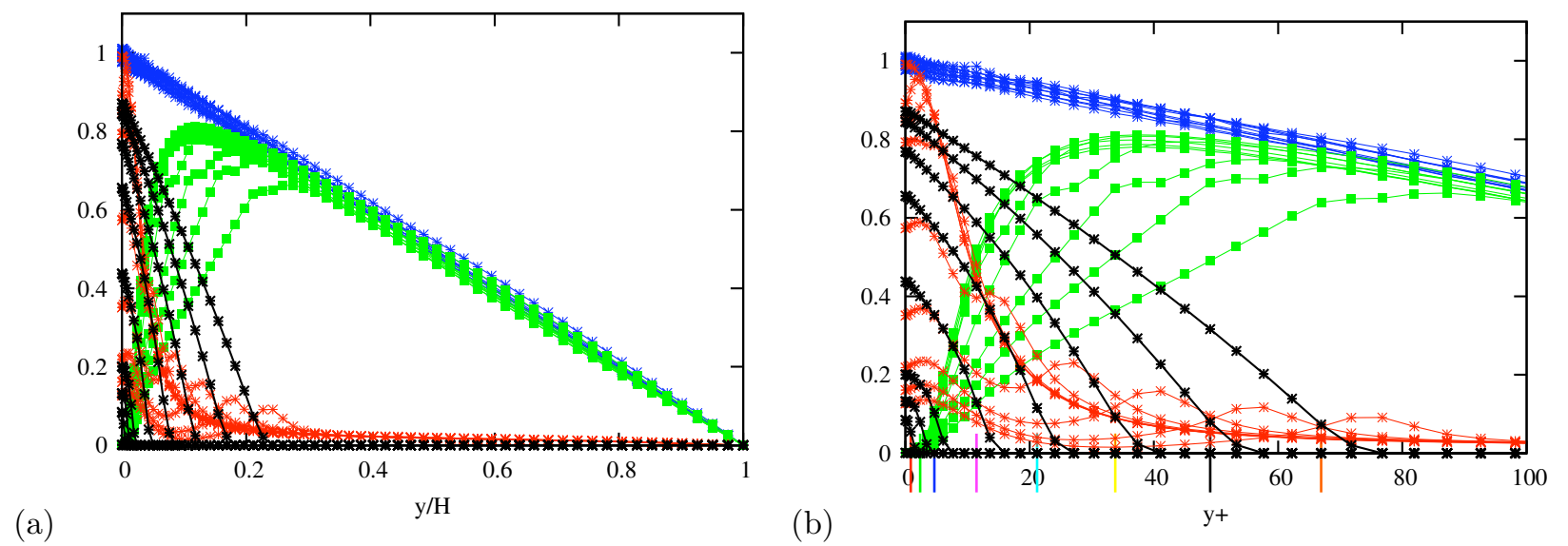

Figure 5. Shear stress decomposition. (a) Outer scaling shows appropriate collapse in the core. (b) Inner scaling shows near wall stress decomposition. The black curve is the roughness induced stress, the green curves are the turbulent shear stress and the red curves are the viscous stress. The total stress in blue the the sum of the others. The stresses are normalized by the wall stress, $\tau_{\text {boundary }}$

\section{III.D. Turbulence kinetic energy production}

Two production terms the balance equation for turbulence kinetic energy, equation II.B. The first is the canonical shear production which represents the conversion of the kinetic energy of the mean flow to turbulent flow. The second is the turbulence production due to the immersed boundary represents the wall roughness. The total production and the production due to Reynolds shear stress are shown in figure 6. The right hand figure shows the production for the low roughness calculations $\left(k^{+}=0-11.46\right)$. For the three smallest peaks in production are the roughness elements less than $5 l^{+}$. Since for these sub-critical roughness heights, the mean velocity and the Reynolds shear stress remain unaffected by the roughness, the shear production of turbulence is not significantly changed. Although for the largest sub-critical roughness the peak in TKE production is due to the roughness, the shear production is still the dominant source.

With increasing roughness heights, the peak of the shear production shifts further from the wall and decreases. The maximum in the production due to the roughness occurs near the crests of the roughness elements. The local maximum in the mean velocity shear leads to a maximum in the shear production peaks at a height above the roughness elements.
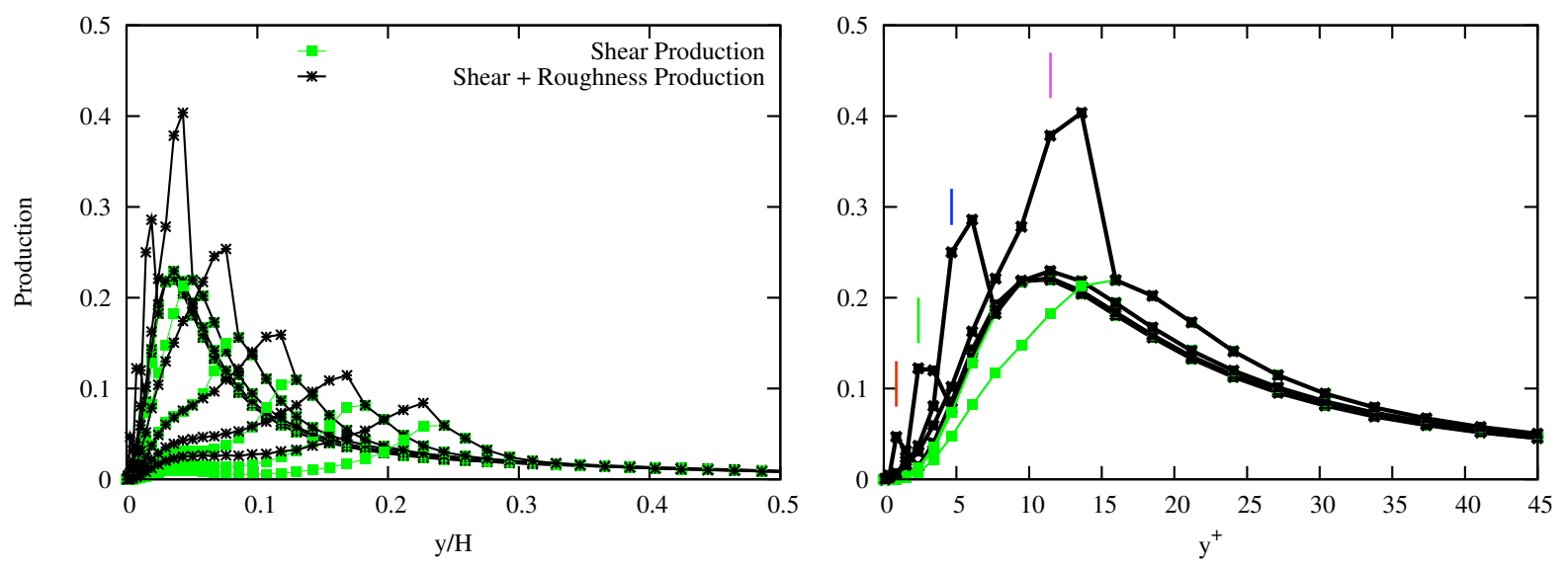

Figure 6. Production of turbulence kinetic energy by mean velocity shear and roughness forcing. 


\section{III.E. Turbulence kinetic energy}

The turbulence kinetic energy profiles exhibits the similar changes with increasing roughness as seen for the production and the mean velocity (figure 7). For the roughness less than the critical height, the turbulence kinetic energy is not altered by the roughness. Although the TKE for the $k^{+}=4.66$ is a slightly increased, neither the location nor the magnitude of the maximum is changed. With increasing roughness the peak in TKE lies close to the tops of the roughness elements and the maximum of the turbulence production. This is in contrast to the canonical case where the maximum in production occurs at approximately $11 l^{+}$, while the maximum of the TKE occurs at approximately $16 l^{+}$. For even the largest roughness elements, the level of TKE normalized by the friction velocity in the core of the channel is largely unaffected by the roughness. The is consistent with the other statistics examined.
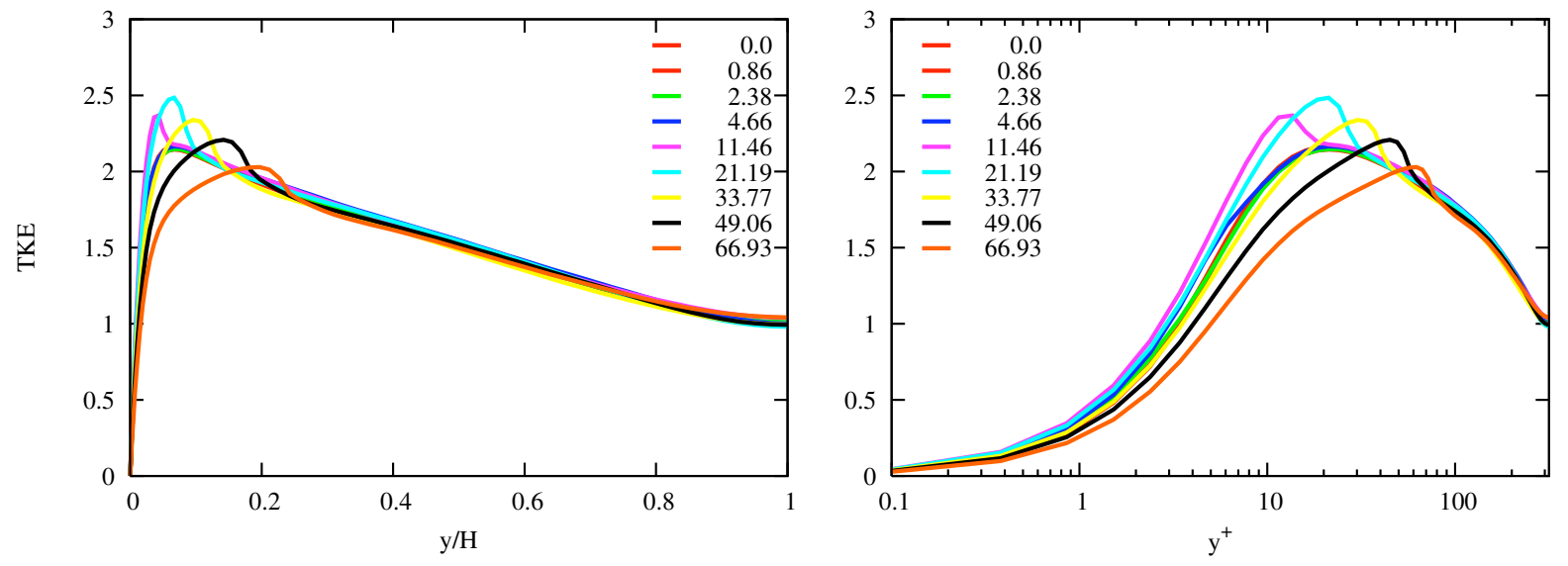

Figure 7. Turbulence kinetic energy.

\section{Conclusion}

The motivation for the research is the development of turbulence models for rough boundaries. The development of turbulence models for rough walled flows usually adopt either an approach of modeling the roughness drag term in the momentum equation or using the modifying the eddy viscosity to predict the combined effect of the turbulent shear stress and the effective roughness stress. The assumption of neglecting the roughness drag or the roughness production is usually made without justification or even discussion. The results present are intended to document that both roughness drag and roughness production play contributing roles.

The partitioning of stress and the production of turbulence kinetic energy into the roughness contributions and the canonical shear contributions has been discussed in the context of DNS results for a range of roughness heights. The results presented above show the development of the near wall turbulence behavior as the roughness height increases. For sub-critical roughness heights, the roughness is shown to have limited effect on the turbulence. Rather the sub-critical roughness increases the form drag while the net viscous drag is decreasing in balance. Although the critical roughness height was not investigated, a transition occurs between $k^{+}=4.66$ and $k^{+}=11.46$ wherein the turbulence production by roughness is significantly increased and the mean velocity begin to decrease.

For even the largest roughness elements, the turbulent core of the channel flow is largely unaffected by the roughness. All turbulent statistics presented converge to the smooth wall statistics just above the roughness elements. Flack, et al. ${ }^{13}$ have attributed this to the geometry of the channel flow and its constraint on the total stress distribution across the channel. In contrast, the mean velocity on the centerline decreases much faster than expected with increasing roughness. This is being attributed to both low Reynolds and to transitional roughness effects, but the cause remains an open question.

The main conclusion from the results presented above is that current approaches to modeling turbulence cannot capture the full effect of roughness. The assumptions used in roughness modeling via augmented eddy viscosity by developers from Cebeci and Chang ${ }^{6}$ to Leighton and Walker, ${ }^{8}$ wherein the roughness drag can 
be neglected, can not be physically justified. Likewise, from the profiles of the Reynolds stress, it is clear that the roughness has a significant affect on the turbulent shear stress. An better modeling approach, alluded to in Leighton and Walker, ${ }^{8}$ is the correct modeling of the mean roughness drag as described in the twophase averaged Navier-Stokes equation and incorporating that model into the full Reynolds stress-transport equation. In this way the roughness can be consistently modeled in both equations.

Future work includes extending the range of simulations through both increases resolution and more general roughness geometries and by the development of full reynolds stress balances.

\section{Acknowledgments}

The author would like to acknowledge the hospitality of the Department of Naval Architecture and Marine Engineering, The University of Michigan during the execution of this work.

\section{References}

${ }^{1}$ ikeda, T.I. and Durban, P.A. 2007 Direct simulations of a rough-wall channel flow. J. Fluid Mechanics.

${ }^{2}$ Hanjalic, K. and Launder, B. E. 1972 Fully developed asymmetric flow in a plane channel. J. Fluid Mechanics

${ }^{3}$ Bhaganagar, K., Kim, J., and Coleman, G., 2004 Effect of roughness on wall-bounded turbulence, Flow Turbul. Combust.

${ }^{4}$ Schlichting, H. 1979 Boundary-Layer Theory, 7th ed. McGraw-Hill.

${ }^{5}$ Taylor, R.P 1983 A Discrete Element Prediction Approach for Turbulent Flow over Rough Surfaces. Ph.D. Dissertation, Mississippi State University

${ }^{6}$ Cebeci, T. and K.C. Chang 1977 Calculation of Incompressible Rough Wall Boundary-Layer Flows, AIAA J.

${ }^{7}$ Durbin, P.A., et al. 2001 Rough Wall Modification of Two-layer $k-\epsilon$, ASME Trans.

${ }^{8}$ Leighton, R.I. and Walker, D.T. 2007 Reynolds stress modeling for rough wall turbulence: An invited Paper, 37th AIAA Fluid Dynamics Conference and Exhibit, Paper AIAA 2007-4615

${ }^{9}$ Joseph, D.D. 2002 Interrogations of Direct Numerical Simulation of Solid-Liquid Flows. http://efluids.com/efluids/books/Interog-1.pdf.

${ }^{10}$ Drew, D.A. 1983 Mathematical Modeling of Two-Phase Flow, Annual Review of Fluid Mechanics.

${ }^{11}$ Goldstein, D., Handler, R. and Sirovich, L., Modeling a no-slip flow boundary with an external force field. 1993 J. Comp. Physics.

${ }^{12}$ Bradshaw, P. 2000 A note on critical roughness height and transitional roughness. Phys. Fluids

${ }^{13}$ Flack, K., Schultz, M.P. and Volino, R.J., Personal Communication, 2008. 